\title{
Investigation of Relation between EFTB Test and RF Conductive Immunity Test Using BER and Baseband Signal
}

\author{
Nobuo Kuwabara ${ }^{1} \cdot$ Yasuhiro Irie $^{1} \cdot$ Norihito Hirasawa $^{2} \cdot$ Yoshiharu Akiyama $^{2}$
}

\begin{abstract}
High-speed telecommunication systems are influenced by electromagnetic environments because they need a wide bandwidth to transmit signals. Immunity tests of telecommunication equipment are effective for improving its immunity to electromagnetic environments. However, immunity tests are expensive to carry out because there are several different tests. The correlation among the tests should therefore be examined in order to reduce the kinds of tests that are necessary. This paper investigates the correlation between the electrical fast transient/burst (EFTB) test and the radio frequency (RF) conductive immunity test. Imitation equipment was constructed with a balun, and a baseband signal was transmitted from the associated equipment to the imitation equipment. Then, disturbances were applied to the equipment, and the telecommunication quality was evaluated by using the bit error rate (BER). The results from the EFTB test indicated that the BER was less than $6 \times 10^{-5}$ and the value was independent of the peak value. The results from the RF conductive immunity test indicated that the BER was affected by the longitudinal conversion loss (LCL).
\end{abstract}

Key words: EFTB, RF Conductive Immunity, Bit Error Rate, Base Band Signal, Longitudinal Conversion Loss (LCL).

\section{Introduction}

Recent progress in telecommunication technology has increased the importance of transmission quality. However, the immunity of telecommunication systems to electromagnetic environments decreases according to the increase in telecommunication speed because a wide bandwidth is needed to transmit high-speed telecommunication signals. Therefore, the induction mechanism of high-speed telecommunication systems has been studied [1], [2].

Common-mode choke coils are effective for improving the conductive immunity of telecommunication equipment, and many kinds of choke coils have been developed [3], [4]. However, choke coils are costly to use after the equipment has been installed because an engineer must visit the installation location. Conductive immunity tests carried out before installation would be effective in reducing the cost of this countermeasure.

The limits and test methods for the immunity of the telecommunication equipment have been published by international standardization committees such as ITU-T and CISPR [5], [6]. However, there are many kinds of immunity tests because the tests are specific for the environment.
Therefore, it is necessary to reduce the various kinds of tests needed for a certain environment by selecting the test method, the failure criteria, and the test level. Thus, the correlation among the different tests should be investigated. It is difficult to reduce the kinds of tests necessary in a certain case because the mechanism causing a malfunction to occur in a telecommunication system may not be clear. However, it is possible if the mechanism is assumed.

In this paper, we assume that the differential-mode signal converted from the common-mode signal disturbs the telecommunication signal. This is one of the main causes of malfunction in high-speed telecommunication systems [1], [2]. Therefore, the cost of implementing countermeasures can be reduced if the number of malfunctions due to this mechanism is reduced. Imitation equipment constructed with a balun was used for the investigation. The immunity levels by the electrical fast transient/burst (EFTB) waveform and the radio frequency (RF) conductive test waveform were tested using the bit error rate (BER). The common-mode signal waveform was measured using a capacitive voltage probe (CVP), and the longitudinal conversion loss (LCL) of the imitation equipment was also measured. The correlation is discussed based on the investigation results.

\footnotetext{
Manuscript received May 16, 2011 ; revised November 14, 2011. (ID No. 20110516-02J)

${ }^{1}$ Dept. of Electrical Engineering and Electronics, Kyushu Institute of Technology, Japan.

${ }^{2}$ Energy and Environment Systems Laboratories, Nippon Telegraph and Telephone Corporation(NTT), Japan.

Corresponding Author : Nobuo Kuwabara (e-mail : kuwabara.nobuo@buddy.elcs.kyutech.ac.jp)
} 


\section{Induction Mechanism and Experimental Set-up}

\section{2-1 Induction Mechanism}

The induction mechanism assumed in this paper is shown in Fig. 1(a). A disturbance appears between the wires and the ground as the common-mode current, and the current is converted to the differential-mode due to the unbalance of the telecommunication equipment, the telecommunication line, and the associated equipment (AE). The malfunction is generated by an overlap of the telecommunication signal with the differential-mode current.

The induction mechanism from the common-mode to the differential-mode are shown in Fig. 1(b). In this figure, $Z_{1}, Z_{2}$, and $Z_{3}$ represents telecommunication equipment. The common-mode current, $I_{c}$, can be expressed by the currents on the conductors, $I_{1}$ and $I_{2}$.

$$
I_{c}=I_{1}+I_{2}
$$

Differential mode current, $I_{d}$, can also be expressed by $I_{1}$ and $I_{2}$, and this is given by

$$
I_{d}=\frac{I_{1}-I_{2}}{2}
$$

When the telecommunication port of the equipment have an unbalance, the $I_{1}$ is not equal to $I_{2}$. In that case, the

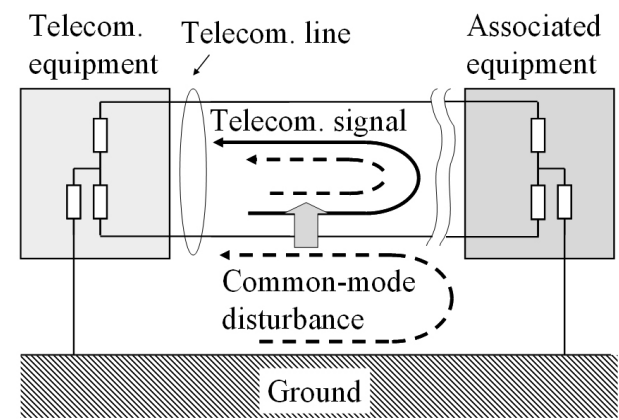

(a) Assumed induction mechanism of malfunction

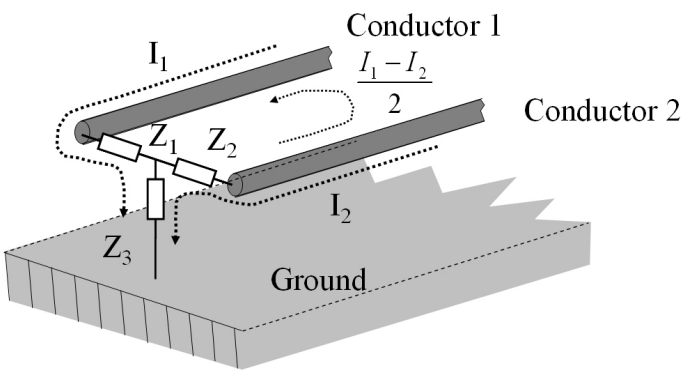

(b) Induction mechanism from common-mode to differential-mode

Fig. 1. Assumed induction mechanism of malfunction and induction mechanism from common-mode to differential mode. differential-mode current is induced as presented by equation (2).

The immunity tests considered for the induction mechanism in Fig. 1(a) are the EFTB test [7], surge immunity test [8], and RF conductive immunity test [9]. Of these, the surge immunity test is not described in this paper because the bandwidth of the frequency spectrum is not wide enough and the occurrence of disturbance is not high enough. Therefore, we investigated the immunity using the EFTB and RF conductive immunity tests.

\section{2-2 Experimental Set-up for EFTB Test}

Fig. 2 shows the experimental set-up for measuring the immunity when the EFTB test waveform is applied. The imitation equipment and the associated equipment were constructed with a balun. An EFTB test waveform generator (NoiseKen FNS-AXII) and the capacitive coupling cramp (NoiseKen 15-00001A) were used to apply the common-mode voltage. The applied common-mode voltage was measured using a CVP (NTT AT, CVP-20S) [10], [11] and an oscilloscope (Yokogawa, DL9040L).

The attenuator (ATT) in Fig. 2 provides the transmission loss of the telecommunication line. Although the loss of an actual telecommunication line changes depending on the frequency, we assume that this loss is independent of the frequency because it is difficult to provide the insertion loss of the telecommunication line. The immunity was evaluated by using a BER measuring instrument (TTC, FIREBERD4000) that uses a baseband signal.

\section{2-3 Experimental Set-up for RF Conductive Immunity Test}

Fig. 3 illustrates the experimental set-up for the RF conductive immunity test. A $2-\mathrm{W}$ coupling decoupling network (CDN) (Hand made) [9], signal generator (R\&S, SMB100A), and amplifier (R\&K, A009K080-5050-R) were used to apply the waveform between the wires and

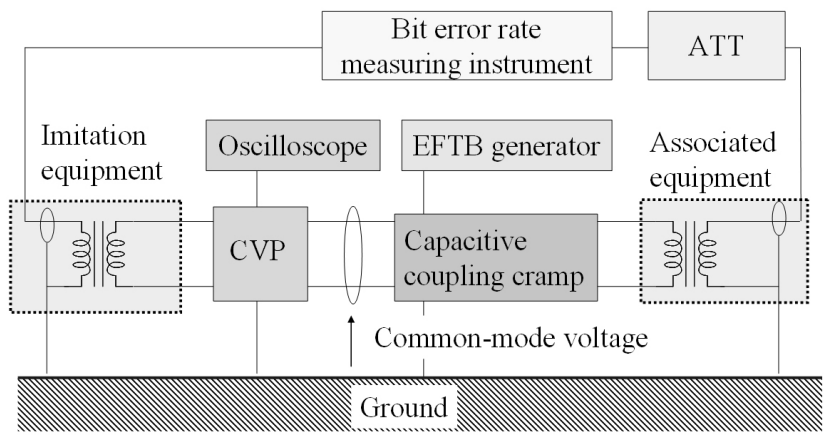

Fig. 2. Experimental set-up for measuring immunity to EFTB test waveform. 


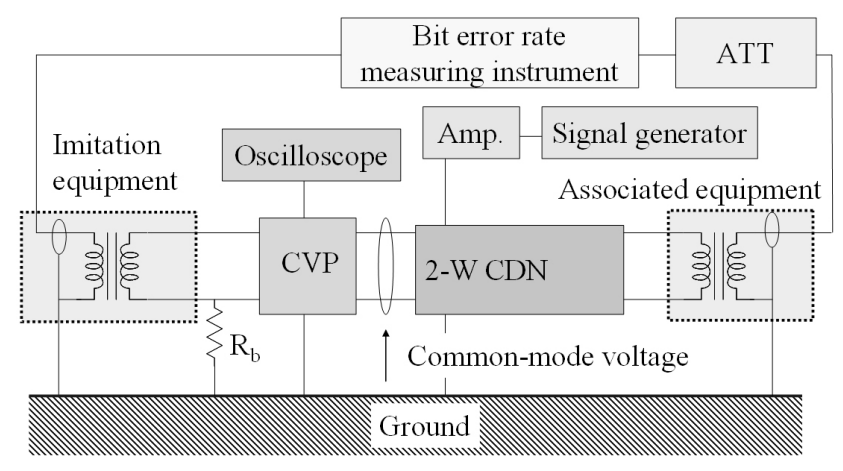

Fig. 3. Experimental set-up for measuring immunity to RF conductive immunity test waveform.

the ground. The amplifier was used to apply a sufficient common-mode level. Its maximum output power was $100 \mathrm{~W}$.

The common-mode voltage was measured by using a CVP and an oscilloscope. A resistance, $\mathrm{R}_{\mathrm{b}}$, was inserted between one wire of the balun and the ground to reduce the longitudinal conversion loss (LCL) [12] of the imitation equipment. LCL means the conversion loss from the common-mode voltage to the differential-mode voltage and this is a parameter to represent a telecommunication system's unbalance about earth.

\section{Investigation Results}

The BER was measured using the experimental set-up shown in Figs. 2 and 3. The layout of the EFTB test is shown in Fig. 4. The EFTB generator, imitation equipment, CVP, and capacitive coupling cramp were placed on the conductive ground plane. A balun whose bandwidth was from $10 \mathrm{kHz}$ to $4 \mathrm{MHz}$ was used as the imitation equipment. The baseband digital signal, whose clock frequency was 2,048 kbps, was used as the telecommunication signal. The waveform of the signal was square.

\section{3-1 Common-mode Voltage}

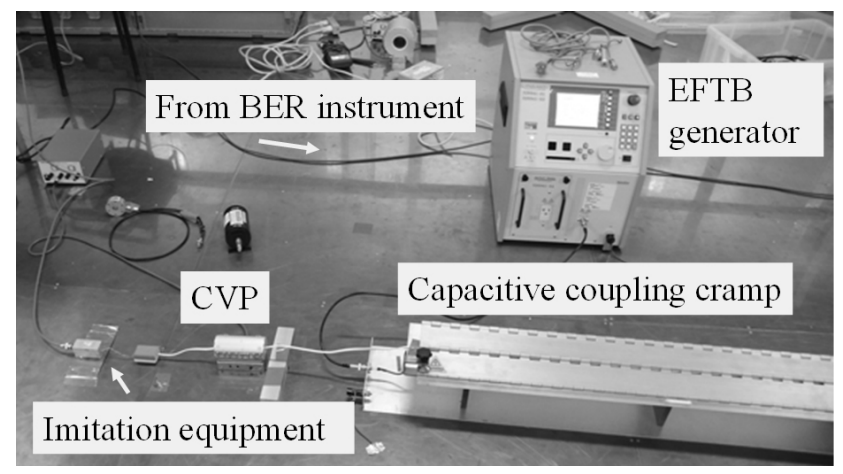

Fig. 4. Layout of experimental set-up for EFTB test.

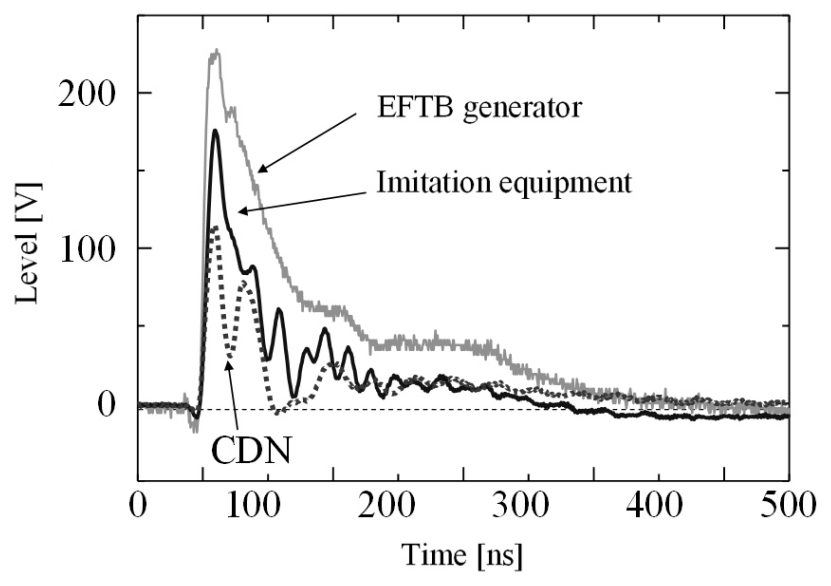

Fig. 5. Measured waveform appearing between wires and ground in EFTB test.

The measured waveform that appeared between the wires and ground in the EFTB test is shown in Fig. 5. In this figure, the "EFTB generator" means the waveform at the EFTB generator output terminal. The waveform is measured by setting the special attenuator at the output terminal in accordance with the IEC publication [7]. "CDN" in Fig. 5 means the waveform measured by the CVP. In this case, the imitation equipment was replaced by the $\mathrm{CDN}$, and the port between the wires and the ground (common-mode port) was terminated by an impedance of $150 \Omega$. This value is lower than that of the imitation equipment. The common-mode voltage waveform of the imitation equipment is similar to the waveform of the EFTB generator. However, the waveform in the case of the CDN is different. This means that the telecommunication line is terminated by a high impedance when the line is terminated by the imitation equipment.

The peak value is reduced by half of the peak value of the EFTB generator when the line is connected to a $\mathrm{CDN}$. In addition, the pulse width of the waveform decreased to one third. These results indicate that the applied waveform is influenced by the common-mode port impedance of the equipment under test (EUT).

Fig. 6 shows a comparison between the common-mode waveform and the differential-mode waveform. The differential-mode waveform was measured at the asymmetrical port of the balun using an oscilloscope. The level of the EFTB generator was $250 \mathrm{~V}$.

The graph shows that a large deviation appears between the common-mode and differential-mode waveforms. This means that the conversion factor from the common-mode voltage to the differential-mode voltage has large frequency dependence.

The measurement results of the LCL for the imitation equipment are plotted in Fig. 7. The LCL was measured according to the definition in the ITU-T recommendation 


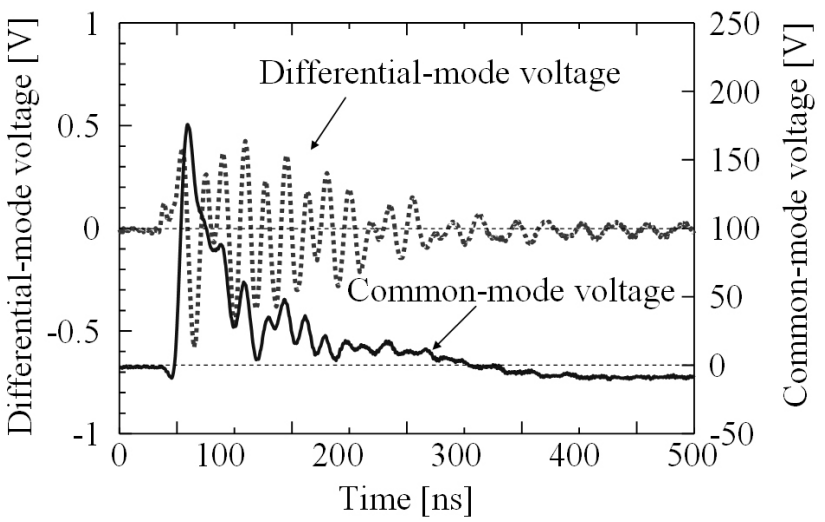

Fig. 6. Comparison between common-mode waveform and differential-mode waveform.

[12]. The probe developed by Macfarlane [13] was used for the measurement.

In this figure, the "LCL probe" means the LCL when the LCL probe port connected to the device under test (DUT) was terminated by a resistance of $100 \Omega$. When the measured value is about the same as the LCL probe value, the measured value is less accurate due to the capacity of the LCL probe. The "imitation equipment (500 $\Omega$ )" refers to the LCL when one of the wires of the imitation equipment was terminated by a resistance of 500 $\Omega$ as represented in Fig. 3.

With the imitation equipment, the LCL value is around $50 \mathrm{~dB}$ in the frequency range from $0.2 \mathrm{MHz}$ to 30 MHz. In the case of the imitation equipment $(500 \Omega)$, the LCL is has almost the same value in the measurement frequency range. This means that the LCL is determined by the resistance of $500 \Omega$.

The peak value of the common-mode voltage was 176 $\mathrm{V}$ in Fig. 6, and that of the differential-mode voltage was $0.58 \mathrm{~V}$ in Fig. 6. The ratio of these voltages was $50 \mathrm{~dB}$. This value is near the LCL value of the imitation equipment in Fig. 7. This means that the waveform appearing at the differential-mode port is influenced by the LCL of the equipment.

The measurement results for the RF conductive immunity waveform are shown in Fig. 8. In this figure, the left vertical axis shows the peak-to-peak voltage level of the waveform. The right vertical axis shows the LCL and the ratio of the common-mode voltage to the differential-mode voltage. The level of the disturbance source is $10 \mathrm{~V}_{\mathrm{rms}}$.

This shows that the differential-mode voltage level, $V_{d}$, increases depending on the frequency increase. On the other hand, the common-mode voltage level, $V_{c}$, does not show a large change. This graph also shows that the ratio of the common-mode voltage to the differential-mode voltage has a similar dependence on the LCL. This means

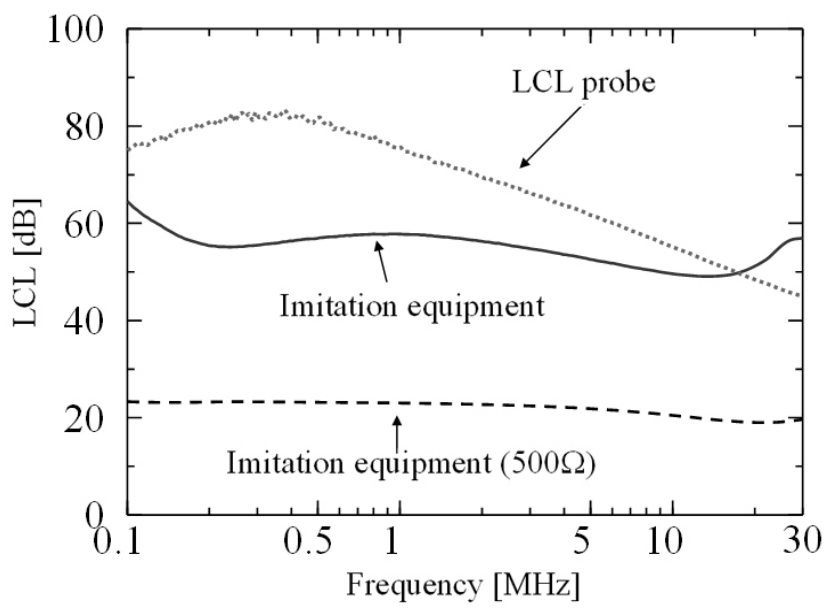

Fig. 7. Results of measuring LCL.

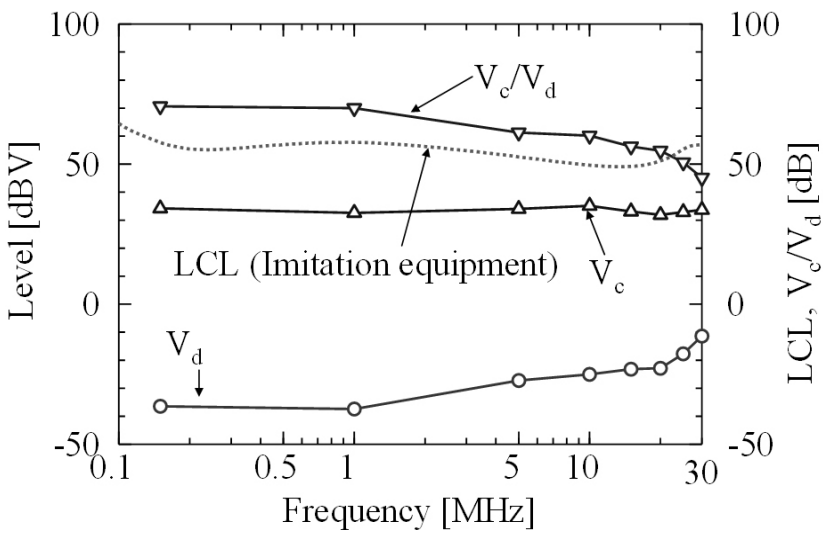

Fig. 8. Measurement results for RF conductive immunity waveform.

that the conversion from the common-mode voltage to the differential-mode voltage is closely related to the LCL.

\section{3-2 Investigation Result of EFTB Test Waveform}

Fig. 9 plots the results of measuring the immunity to the EFTB waveform. In this figure, the horizontal axis is the signal level in $\mathrm{dBV}$, which is represented by the peak value. The vertical axis is the BER. The circles indicate the burst frequency of $100 \mathrm{kHz}$, and the triangles indicate the burst frequency of $5 \mathrm{kHz}$. The squares indicate the BER when no disturbance was applied. Here, the burst frequency means the period of the surge waveform. The white circles and triangles represent the peak value of $250 \mathrm{~V}$, and the solid circles and triangles represent the peak value of $500 \mathrm{~V}$.

First, we consider the case where the peak value is $250 \mathrm{~V}$. When the signal level was less than $-13 \mathrm{dBV}$, the BER mainly depended on the system noise of the in- 


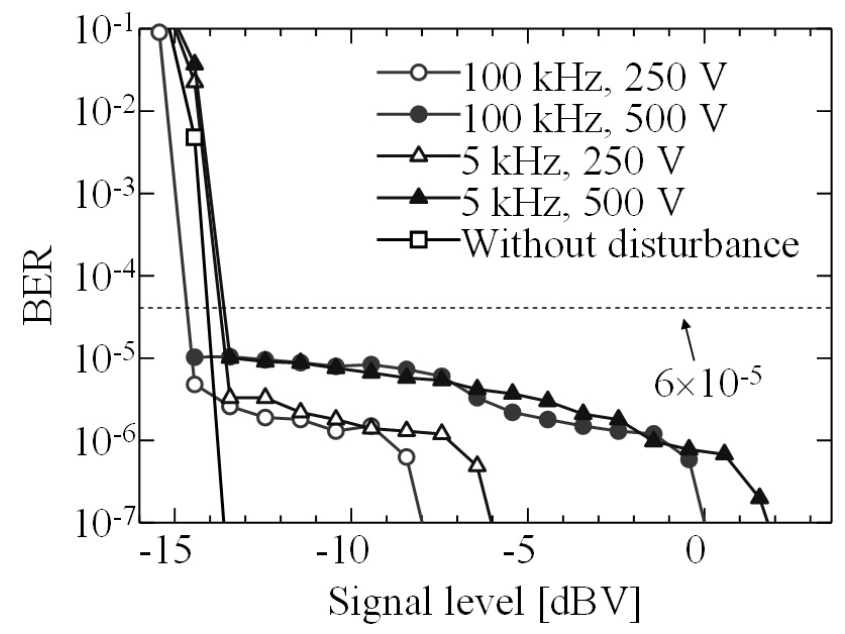

Fig. 9. Measured BER for EFTB waveform.

strument used to measure BER. On the other hand, when the signal level was more than $-13 \mathrm{dBV}$, the BER mainly depended on the applied disturbances. The BER initially decreased, which is shown as a gentle slope; it decreased rapidly when the signal level was more than $-7 \mathrm{dBV}$. This value almost agrees with the peak value of the disturbances between wires.

We next consider the case where the peak value is 500 $\mathrm{V}$. When the signal level was less than $-13 \mathrm{dBV}$, the BER mainly depended on the system noise of the BER measuring instrument, similar to the $250 \mathrm{~V}$ case. The BER decreased, represented as a gentle slope, when the signal level was more than $-13 \mathrm{dBV}$, and then it decreased rapidly when the signal level was more than $1 \mathrm{dBV}$. This value is $8 \mathrm{~dB}$ larger than the $250 \mathrm{~V}$ case and is similar to the ratio of $500 / 250(6 \mathrm{~dB})$.

No significant difference appears between the burst frequency of $100 \mathrm{kHz}$ and the burst frequency of $5 \mathrm{kHz}$. Fig. 10 shows the differential-mode waveform measured

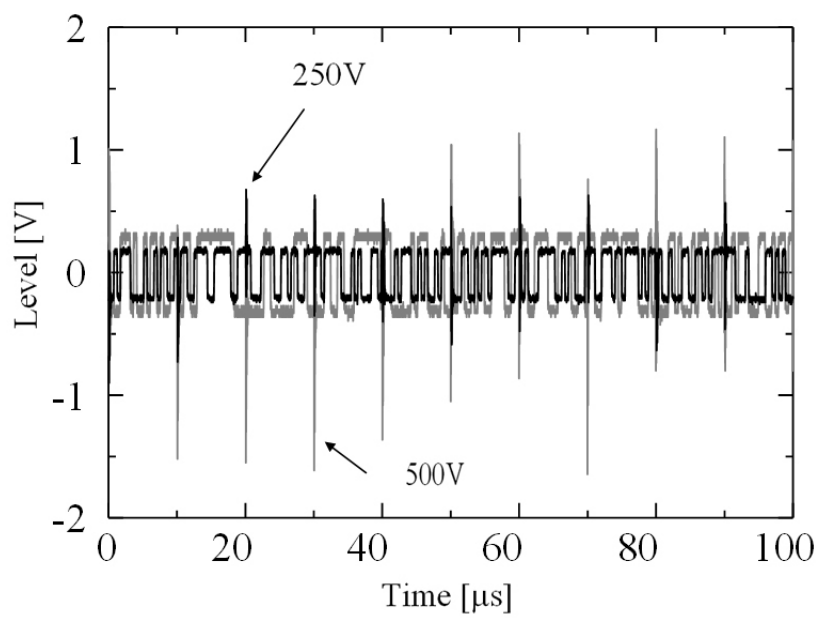

Fig. 10. Measured BER for EFTB waveform. at the unbalance port of the balun. The black line indicates the waveform when the peak value is $250 \mathrm{~V}$, and the gray line indicates the waveform when the peak value is $500 \mathrm{~V}$. This shows that the level of the EFTB pulse is larger than the signal level. This means the bit error appears when the disturbance is applied to the telecommunication signal.

The investigation result in Fig. 10 suggests that the BER can be calculated from the probability where the peak value exceeds the signal level. Based on that assumption, the BER can be calculated from the following equation.

$$
B E R=0.5 \frac{n}{N}=0.5 \frac{n}{f / T}
$$

Here, $n$ is the number of bursts, $f$ is the clock frequency of the signal, and $T$ is the burst period. The value of 0.5 indicates the probability that the bit error will occur when the signal level is low.

When $\mathrm{n}$ is $75, f$ is $2,048 \mathrm{kHz}$, and $T$ is $300 \mathrm{~ms}$, the BER is $6 \times 10^{-5}$. This value is also shown in Fig. 9. This means that the BER can be roughly estimated from the probability where the disturbance level exceeds the signal level. In addition, the BER is less than $6 \times 10^{-5}$ when the peak value is any large value. Equation (1) suggests that the BER is dependent on the number of bursts.

\section{3-3 Investigation Results of RF Conductive Immunity Test}

Fig. 11 shows the investigation results for the RF conductive immunity test. Carrier frequencies of 0.15 and 1 , $5,10,15,20,25$, and $30 \mathrm{MHz}$ were selected for the experiment. The level of the disturbance source was 20 $\mathrm{V}_{\text {rms }}$ (the peak-to-peak value was $100 \mathrm{~V}$ ). "N. D." means the measurement results when the disturbance level was zero.

The BER maintains a value around $1 \times 10^{-2}$ when the signal level is lower than a threshold value. The BER decreases rapidly when the level exceeds the value. The threshold level changes depending on the carrier frequency. It is low at a low carrier frequency. This means that the converted differential mode is low as shown in Fig. 11. The maximum level is at $5 \mathrm{MHz}$, and this decreased depending on the increase in frequency. This is caused by the frequency characteristics of the balun because the frequency bandwidth is from $10 \mathrm{kHz}$ to 4 $\mathrm{MHz}$.

Fig. 12 shows the BER when one of the imitation equipment terminals is connected to the ground via the resistance $R_{b}$. The $R_{b}$ of $500 \Omega$ was selected for the experiment. The disturbance source level was $10 \mathrm{~V}_{\mathrm{rms}}$ (the peak-to-peak value was $50 \mathrm{~V}$ ). 


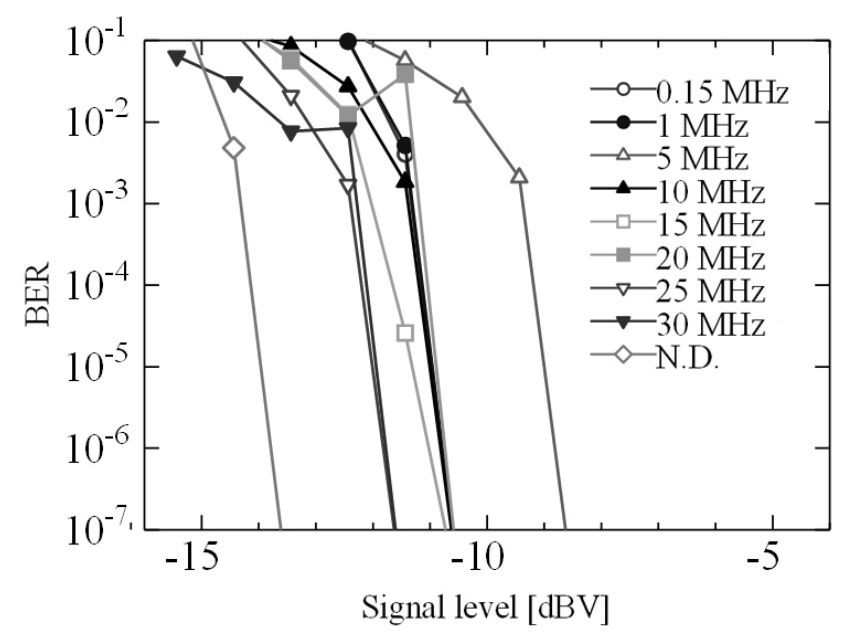

Fig. 11. Measured BER for RF conductive immunity test.

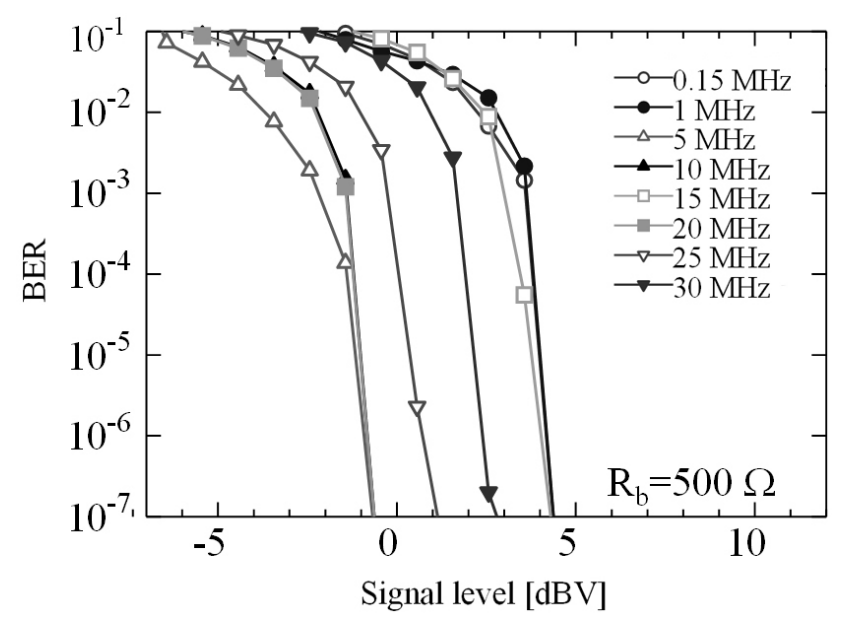

Fig. 12. Measured BER for RF conductive immunity test when $R_{b}$ is $500 \Omega$.

The threshold level to which the BER drops rapidly is increased by inserting resistance. For example, the value increases from -11 to $3 \mathrm{dBV}$ at $0.15 \mathrm{MHz}$. This causes that the LCL of the imitation equipment decreases when resistance is inserted.

When the carrier frequency is more than $20 \mathrm{MHz}$, the threshold level is smaller than that when the frequency is less than $5 \mathrm{MHz}$ because of the frequency characteristics of the balun.

\section{3-4 Correlation between EFTB Test and RF Conduc- tive Immunity Test}

With the EFTB test, the BER is affected when the signal level is less than $1 \mathrm{dBV}$. In this case, the peak value of the differential-mode voltage (telecommunication signal) is estimated to be $1.16 \mathrm{~V}$ from Fig. 6. With the RF conductive immunity test, the BER is affected when the signal level is less than $-10 \mathrm{dBV}$ for the frequency of $5 \mathrm{MHz}$. At that time, the differential-mode voltage of disturbances is determined to be $-21 \mathrm{dBV}((-27+6)$ $\mathrm{dBV}$ ) from Fig. 8, where the value of 6 is the conversion factor from the unbalance port of the balun to the balance port. In this case, the zero-peak value of telecommunication signal is $0.045 \mathrm{~V}$ and the ratio of $1.16 \mathrm{~V}$ to $0.045 \mathrm{~V}$ is $28 \mathrm{~dB}$.

The investigation results indicate that the BER increases when the disturbance appears at the differentialmode. Based on this result, the RF conductive immunity test is more severe than the EFTB test if the zero-peak value is the same. However, the test level of the EFTB test was higher than that of the RF conductive immunity test. Therefore, the EFTB test is more severe than the RF conductive immunity test.

However, the maximum BER of the EFTB test was less than $6 \times 10^{-5}$. Therefore, the RF conductive immunity test was found to be more severe than the EFTB test if the telecommunication system is not affected by the BER value.

\section{IV . Conclusion}

The correlation between the EFTB test and the RF conductive test was investigated. In the investigation, we assumed that a malfunction of the telecommunication system occurs due to differential-mode disturbance that is converted from common-mode disturbance.

The BER was used to evaluate the degree of immunity level to the disturbances. The common-mode waveform was measured using a CVP. The measurement results of the common-mode waveform by the EFTB test show that the waveform changes in the telecommunication equipment. The LCL of the imitation equipment was measured, and the results showed that the LCL was around $50 \mathrm{~dB}$, and this is decreased by connecting one wire to the ground via a resistance.

The investigation showed that the ratio of the common-mode voltage to the differential-mode voltage was influenced by the LCL. The results of measuring the common-mode voltage in the RF conductive immunity test also showed that the ratio of the common-mode voltage to the differential-mode voltage had a similar value to the LCL.

The investigation using the EFTB test indicated that the maximum BER was less than $6 \times 10^{-5}$. The investigation using the RF conductive immunity test indicated that the BER was closely related to the LCL. These results suggest that the EFTB test is more severe than the RF conductive immunity test if a malfunction occurs due to the mechanism assumed in this paper. 
In this paper, we assumed that the telecommunication signal was disturbed by the induced differential-mode current from common-mode current. However, the evaluation to many telecommunication equipment are required to clarify the correlation between immunity tests because another induction mechanism may exist.

The authors thank Dr. M. Matsuoka for his support of this study.

\section{References}

[1] K. Takaya, H. Yoshioka, K. Mokushi, and M. Toyonaga, "Influence of differential configurations of premises cable on the interference immunity of VDSL system," International Symposium on EMC, Kyoto, 22R2-4, pp. 369-372, Jul. 2009.

[2] N. Nakamura, R. Kobayashi, "Immunity test of IP devices against repetitive impulsive noise," International Symposium on EMC, Kyoto, 21P3-2, pp. 129132, Jul. 2009.

[3] K. Takagi, H. Tanaka, A. Kuriyama, and S. Shinohara, "Electromagnetic disturbances protection for telecommunications terminal equipment," NTT Review, vol. 5, no. 3, pp. 49-52, May 1993.

[4] F. Amemiya, K. Takagi, N. Kuwabara, S. Hamada, and Y. Iwamoto, "Developing common-mode choke coil with high-permeability core used high-speed telecommunication port for UTP cable," IEEE International Symposium on EMC, Minneapolis, pp. 314319, Aug. 2002.

[5] IEC/CISPR Publication 24, "Information technology

Nobuo Kuwabara

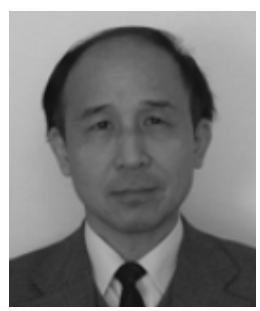

received his B.E. and M.E. degrees in electronic engineering from Shizuoka University in 1975 and 1977, respectively. He received a D.E. degree from Shizuoka University in 1992. He joined NTT in 1977, where he was initially engaged in research on overvoltage protection and electromagnetic compatibility relating to telecommunication system. Since April, 2001, he has been a professor in the Department of Electrical Engineering and Electronics at Kyushu Institute of Technology, where he is continuing the research on electromagnetic compatibility. He is a member of the IEICE, the IEEE, and the IEEJ. equipment - Immunity characteristics - Limits and methods of measurement," Sep. 1997.

[6] ITU-T K.43, "Immunity requirements for telecommunication equipment," May 1998.

[7] IEC61000-4-4, "Electromagnetic compatibility (EMC) - Part 4: Testing and measurement techniques - Section 4: Electrical fast transient / burst immunity test - Basic EMC Publication," 1995.

[8] IEC61000-4-5, "Electromagnetic compatibility (EMC) - Part 4: Testing and measurement techniques Section 5: Surge immunity tests - Basic EMC Publication," 1995.

[9] IEC61000-4-6, "Electromagnetic compatibility (EMC) - Part 4: Testing and measurement techniques - Section 6: Immunity to conducted disturbances, induced by radio-frequency fields," 1996.

[10] IEC/CISPR Publication 16-1-2 Edition 1.2, "Specification for radio disturbance and immunity measuring apparatus and method - Part 1-2: Radio disturbance and immunity measuring apparatus - Ancillary equipment - Conducted disturbances," Aug. 2006.

[11] R. Kobayashi, Y. Hiroshima, H. Ito, H. Furuya, M. Hattori, and Y. Tada, "A novel non-contact capacitive probe for common-mode voltage measurement," IEICE Trans. Commun., vol. E90-B, no. 6, pp. 1329-1337, Jun. 2007.

[12] ITU-T G. 117, "Transmission aspects of unbalance about earth," Feb. 1996.

[13] N. Oka, A. Konemori, Y Sakai, H. Fukushima, M. Kato, and S. Nitta, "Radiated emission from PLC and electrical unbalance of an artificial power distribution line," International Symposium on EMC, Sendai, 4C3-2, pp. 841-844, Jun. 2004.

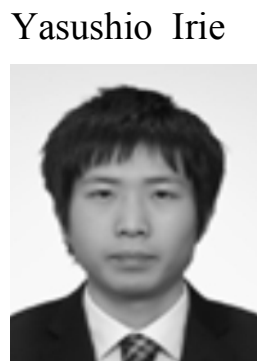

is a graduate student at Kyushu Institute of Technology, where he has been studying the relations among immunity tests since April, 2010. 


\section{Norihito Hirasawa}

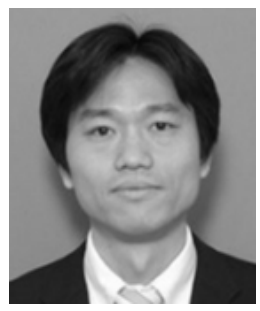

received a B.E. degree in electrical engineering from the Tokyo University of Science, Japan in 1998. He joined the NTT Electrical Telecommunication Laboratory, NTT Corporation in 1998. From 2001 to 2008 , he worked at the corporate business headquarters of NTT East Corporation, and in 2008 he joined the NTT Energy and Environment Systems Laboratories. He has been researching EMC of broadband communications such as power line telecommunications.

\section{Yoshiharu Akiyama}

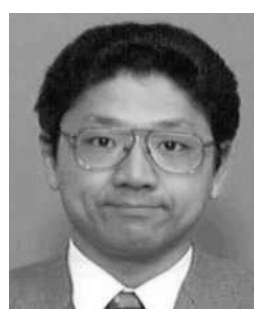

received his B.E. and D.E. degrees from University of Electro Communications in 1990 and 2010, respectively. Since joining NTT he has been researching methods of radiated electromagnetic field immunity testing, EMC on wireless LAN, broadband communication systems such as XDSL and PLC, and methods of shield effectiveness measurement. $\mathrm{He}$ is a senior researcher and leader of the EMC Technology Group. 